\title{
Safety of Hydrochlorothiazide in the Real World: A Commentary on the Italian Medicines Agency (AIFA) Pronouncements
}

\section{Valerio Massimo Magro, $M D^{1^{*}}$, Carla Coppola, $M D^{2}$, Eugenio Boccalone, $M D^{1}$, Michele Caturano, $M D^{3}$, Giovanni Scala, $M D^{4}$ and Walter Verrusio, MD, $P h D^{5}$}

${ }^{1}$ Geriatrician, Department of Internal Medicine and Geriatry, University of Campania Luigi Vanvitelli, Italy

${ }^{2}$ Geriatrician, Department of Intensive Rehabilitation, Casa di Cura Alma Mater S.p.A, Villa Camaldoli, Italy

${ }^{3}$ Department of Internal Medicine, AORN San Giovanni di Dio e Ruggi d'Aragona, Italy

${ }^{4}$ Geriatrician, Doctor Responsible for Home Care Center (CAD), RM2 ASL, Italy

${ }^{5}$ Geriatrician, Department of Cardiovascular, Respiratory, Nephrological, Anesthesiological and Geriatric Sciences, Sapienza University of Rome, Italy

*Corresponding author: Valerio Massimo Magro, MD, Geriatrician, Department of Internal Medicine and Geriatry, University of Campania Luigi Vanvitelli, Piazza L. Miraglia 2, 80100, Naples, Italy, Tel: 3492224922

\section{Background}

Thiazide diuretic drugs are the most commonly used molecules for the treatment of systemic hypertension. Despite their established role in the treatment of this disease, well recognized by the guidelines of national (such as the Italian Society of Cardiology - SIC - for example, which incorporates the directives of the European Society of Cardiology - ESC -) scientific societies $[1,2]$ - including those that operate specifically in the setting of the General Medicine $[3,4]$ - that international scientific societies [58], with recognized effect and good class of recommendation, nevertheless in recent times new advice has arisen from studies present in the literature that have undermined its safety and warned the clinician of the dangers concerning their use. In particular, our attention focused on a molecule such as hydrochlorothiazide.

\section{Known Adverse Events of Hydrochlorothiazide}

Several evidences have shown that hydrochlorothiazide therapy in chronic renal disease with advanced grade of kidney insufficiency leads to deleterious effects, as the adverse effects of this therapy are reported (worsening of renal function, depletion of water volume, hyponatremia, hypercalcemia, hyperglycemia, dyslipidemia) [9]. Regarding the electrolyte imbalances, thiazide-induced hyponatremia is certainly an important medical condition, that is regularly present in the internal medicine departments and it causes substantial morbidity to frail patients, often elderly, who are taking hydrochlorothiazide, in subgroups of patients $[10,11]$. Equally not yet well understood is the mechanism by which hydrochlorothiazide interferes with the glucose homeostasis [12-14]. There is also a whole range of side effects, fortunately rarer, but described in the medical literature, ranging from the appearance of lupus [15], interstitial lung disease [16], even severe allergic states [17], pulmonary edema [18-20], all conditions linked to immunological changes induced by the molecule itself. The effect on the sense organs (hypoacusis) is more known and less rare (although investigated with low frequency, especially in the elderly subjects), with various reports from the organs of vigilance drug, for which there are well defined indications for the diagnosis of this adverse event (Austin Bradford-Hill criteria, Naranjo criteria), well applied in pharmacovigilance [21,22].

\section{Pharmacovigilance in Italy}

With regard to this aspect, it is worth mentioning 
a recent intense vigilance drug activity for cutaneous hydrochlorothiazide adverse events. We recall that pharmacovigilance is the set of activities whose objective is to provide, on an ongoing basis, the best possible information on the safety of medicines, thus allowing the adoption of appropriate measures and in this way ensuring that the drugs available on the market, in the conditions of authorized use, a favorable benefit risk ratio for the population. There is a "Pharmacovigilance" section of the Italian Drug Agency (Agenzia Italiana del Farmaco - AIFA) dedicated to General Practitioners (GPs) and to the Pediatricians. The service is bidirectional, i.e., the GPs/Pediatricians can (directly) send the adverse drug reaction (ADR) report to the pharmacovigilance network of AIFA, that will insert it within the scheduled time on the National Pharmacovigilance Network. In the opposite direction, the doctor will be able to receive letters by mail from the body (a collection of such letters exists on the site of AIFA http://www.agenziafarmaco.gov. $\mathrm{it} / \mathrm{it} / \mathrm{nii})$. Towards the end of 2018, AIFA sends one of these letters to doctors about hydrochlorothiazide and its skin effects [23]. Also the latter have long been reported in the literature and very heterogeneous from the clinical point of view, going from lichenoid dermatitis to skin eruptions similar to pityriasis rosea to erythema multiforme to the vasculitic reaction [24-27]. Recently, however, the attention of epidemiologists has focused on drug-induced oncogenesis. The photosensitizing action of hydrochlorothiazide, in fact, combined with the generation of free radicals, is known to have a damaging action on the skin in a cancerizing sense [28]. Oxygen free radicals, in fact, generated by exposure to ultraviolet rays and enhanced by hydrochlorothiazide, cause DNA damage with alterations of nitrogenous bases and formation of thymine dimers that have cells on the skin, active labile cells replication, an oncogenic effect $[29,30]$. In addition to the skin squamous cell carcinoma, in the year 2013 a study put this drug in relation with other skin cancers, such as the cutaneous T-cell lymphomas [31], without clarifying a true causal link but postulating a possible genotoxic action, similar to that on other skin cells [30]. A more recent Danish study, investigating the non-melanomatous tumors in the Danish Cancer Registry, focusing on over 70,000 cases of basal cell carcinoma and over 8,600 patients with squamous cell carcinoma, respectively compared with control populations of over $1,400.000$ and over 170,000 subjects, found that the high use of hydrochlorothiazide (at the cumulative dose equal to $50,000 \mathrm{mg}$ - corresponding to $12.5 \mathrm{mg}$ of this drug taken daily for about 11-years or higher) was associated with an adjusted odds ratio (OR) to 1.29 (with a $95 \%$ confidence interval $-\mathrm{Cl}-1.23-1.35)$ for basal cell carcinoma and 3.98 (95\% Cl: 3.68-4.31) for carcinoma a squamous cells, with a cumulative dose-response relationship for both of these tumor forms [32]. The use of other types of diuretic and other antihypertensive drugs was not associated with non-melanomatous skin tumors. Another study showed a possible association between labial squamous cell carcinoma and the exposure to this thiazide, in 633 cases compared to 63,067 controls, with a cumulative dose-response relationship with adjusted OR of 2.1 (95\% Cl : 1.7-2.6) which increased up to OR 3.9 (3.0-4.9) for patients who used it at high dose ( $25.000 \mathrm{mg}$ ) and a OR 7.7 (5.7-10.5) for the highest cumulative dose ( $100,000 \mathrm{mg}$ ) [33]. Finally, a recent study, conducted with the evaluation of different databases (PubMed, EMBASE, Cochrane Library), would confirm the association with increased risk of skin cancers, especially squamous cell carcinoma (underlining the need for further studies to confirm the results) [34]. The AIFA report concluded the report by inviting the physicians to the following prescriptive behaviors and to adopt the following precautions: information on the risk of non-melanoma skin cancers for patients taking hydrochlorothiazide, alone or in combination with other drugs, with the regular control and self-control of the skin for the early identification of any new lesions or changes to existing lesions, with notification to your doctor of any suspected skin lesion; examination of suspected skin lesions both from a clinical but also histological point of view, using the bioptic exam; warning patients about the limitation of exposure to sunlight/UV rays, with the use of adequate protection when exposed to sunlight/UV rays, to minimize the risk of skin cancers; careful evaluation and discernment on the prescription/use of hydrochlorothiazide in patients who have had a previous skin cancer.

\section{Conclusions}

Recent studies showed the presence of a cumulative dose-dependent association between hydrochlorothiazide and non-melanoma skin cancers. There was a statement with an information note issued by the European/Italian monitoring and surveillance organizations (European Medicines Agency - EMA - and AIFA). There is a need for the integration of pharmacological-epidemiological data and those data coming from the registers, the various databases and the studies already present in the literature with data collected in the real world, both in the academic environment but even more so in the field, especially from the databases in possession of general practitioners, to have as far as possible an objective picture of the situation.

\section{Contributors}

Magro VM was the primary researchers and wrote the manuscript. Coppola C, Caturano M, Boccalone E and Scala $G$ provided research and editing assistance to the manuscript. Magro VM, Coppola C, Caturano M, Boccalone E, Scala G and Verrusio W contributed to overall article design, data collection as well as revising 
and approving the manuscript.

\section{Conflict Interest and Disclosures}

None declared.

\section{References}

1. (2018) ESC/ESH 2018 guidelines for the diagnosis and treatment of arterial hypertension. Task force for diagnosis and treatment of arterial hypertension of the European Society of Cardiology (ESC) and the European Society of Arterial Hypertension (ESH). G Ital Cardiol 19: 3S-73S.

2. Macia G, Fagard R, Narkiewicz K (2013) ESH/ESC 2013 guidelines for the diagnosis and treatment of high blood pressure. Task force for diagnosis and treatment of arterial hypertension of the European Society of Arterial Hypertension (ESH) and of the European Society of Cardiology (ESC). Ipertensione 20: 1-73.

3. Battingelli D, Bettoncelli G, D'Ambrosio G (2014) Guide to Hypertension. Italian Society of General Medicine (SIMG) - Italian Society of Arterial Hypertension (Società Italiana dell'Ipertensione Arteriosa, SIIA).

4. Contursi V, Zaninelli A, Maso G (2016) Practical Handbook for the management of Arterial Hypertension in General Medicine. Italian Journal of Primary Care 6.

5. Whelton PK, Carey RM, Aronow WS, Karen J Collins, Cheryl Renee Dennison-Himmelfarb, et al. (2018) 2017 ACC/ AHA/AAPA/ABC/ACPM/AGS/APhA/ASH/ASPC/NMA/ PCNA Guideline for the Prevention, Detection, Evaluation, and Management of High Blood Pressure in Adults. Journal of the American College of Cardiology 71: e127-e248.

6. Tamargo J, Segura J, Ruilope LM (2014) Diuretics in the treatment of hypertension. Part 1: thiazide and thiazide-like diuretics. Expert Opin Pharmacother 15: 527-547.

7. Vongpatanasin W (2014) Resistant hypertension: A review of diagnosis and management. JAMA 311: 2216-2224.

8. Gauer R (2017) Severe Asymptomatic Hypertension: Evaluation and Treatment. Am Fam Physician 95: 492-500.

9. Pourafshar N, Alshahrani S, Karimi A (2018) Current Drug Metabolism. 19: 1012-1020.

10. Nadal J, Channavaijhala SK, Jia W, Clayton J, Hall IP, et al (2018) Clinical and molecular features of thiazide-induced hyponatremia. Curr Hypertens Rep 20: 31.

11. Glover M, Clayton J (2012) Thiazide-induced hyponatraemia: Epidemiology and clues to pathogenesis. Cardiovasc Ther 30: e219-e226.

12. Price AL, Lingvay I, Szczepaniak EW, Wiebel J, Victor RG, et al. (2013) The metabolic cost of lowering blood pressure with hydrochlorothiazide. Diabetol Metab Syndr 5: 35.

13. Thomopoulos C, Tsioufis C, Makris T, Stefanadis C (2011) The 'pyrrhic victory' of amlodipine over hydrochlorothiazide in the OLAS Study. J Hum Hypertens 25: 401-402.

14. Palming J, Jansson PA, Renström F, Johansson A, Johansson L, et al. (2011) Hydrochlorothiazide compared to candesartan treatment increases adipose tissue gene expression and circulating levels of serum amyloid $A$ in hypertensive patients. Horm Metab Res 43: 319-324.

15. Sosenko T, Pasula S, Brahmamdam R, Girnita D (2019) When Chest Pain Reveals More: A Case of Hydrochlorothiazide-Induced Systemic Lupus Erythematosus. Am J Case Rep 20: 26-30.

16. Biron P, Dessureault J, Napke E (1991) Acute allergic interstitial pneumonitis induced by hydrochlorothiazide. CMAJ 145: $28-34$.
17. Mineo MC, Cheng EY (2009) Severe allergic reaction to hydrochlorothiazide mimicking septic shock. Pharmacotherapy 29: 357-361.

18. Knowles SR, Wong GA, Rahim SA, Binkley K, Phillips EJ, et al. (2005) Hydrochlorothiazide-induced noncardiogenic pulmonary edema: An underrecognized yet serious adverse drug reaction. Pharmacotherapy 25: 1258-1265.

19. Goetschalckx K, Ceuppens J, Van Mieghem W (2007) Hydrochlorothiazide-associated noncardiogenic pulmonary oedema and shock: A case report and review of the literature. Acta Cardiol 62: 215-220.

20. Young CC, Kyle JL, Young RT (1997) Acute pulmonary edema caused by ingestion of hydrochlorothiazide. Wis Med J 96: 38-39.

21. Belai N, Gebrehiwet S, Fitsum Y, Russom M (2018) Hydrochlorothiazide and risk of hearing disorder: A case series. $J$ Med Case Rep 12: 135.

22. Shakir SA, Layton D (2002) Causal association in pharmacovigilance and pharmacoepidemiology: Thoughts on the application of the Austin Bradford-Hill criteria. Drug Saf 25: 467-471.

23. http://www.quotidianosanita.it/allegati/allegato8646489.pdf

24. Ruscin JM, Page RL 2nd, Scott J (2006) Hydrochlorothiazide-induced angioedema in a patient allergic to sulfonamide antibiotics: Evidence from a case report and a review of the literature. Am J Geriatr Pharmacother 4: 325-329.

25. Atzori L, Pinna AL, Ferreli C, Aste N (2006) Pityriasis rosea-like adverse reaction: Review of the literature and experience of an Italian drugsurveillance center. Dermatol Online J 12: 1.

26. Petavy-Catala C, Martin L, Fonte`s V, Lorette G, Vaillant L (2001) Hydrochlorothiazide-induced acute generalized exanthematous pustulosis. Acta Derm Venereol 81: 209.

27. Burckhardt W, Sutter T (1963) Photoallergic drug exanthema caused by hydrochlorothiazide. Z Haut Geschlechtskr 34: 105-108.

28. Campione E, Di Prete M, Diluvio L, Bianchi L, Orlandi A (2016) Efficacy of ingenol mebutate gel for actinic keratosis in patients treated by thiazide diuretics. Clin Cosmet Investig Dermatol 9: 405-409.

29. Kunisada M, Masaki T, Ono R, Morinaga H, Nakano E, et al. (2013) Hydrochlorothiazide enhances UVA-induced DNA damage. Photochem Photobiol 89: 649-654.

30. Alzoubi KH, Bayraktar E, Khabour O, Sayer I.Al-Azzam (2018) Vitamin B12 protects against DNA damage induced by hydrochlorothiazide. Saudi Pharmaceutical Journal 26: 786-789.

31. Jahan-Tigh RR, Huen AO, Lee GL, Pozadzides JV, Liu P, et al. (2013) Hydrochlorothiazide and cutaneous T cell lymphoma: prospective analysis and case series. Cancer 119: 825-831.

32. Pedersen, Gaist D, Schmidt SAJ, Hölmich LR, Friis S, et al. (2018) Hydrochlorothiazide use and risk of nonmelanoma skin cancer: A nationwide case-control study from Denmark. J Am Acad Dermatol 78: 673-681

33. Pottegard A, Hallas J, Olesen M, Svendsen MT, Habel LA, et al. (2017) Hydrochlorothiazide use is strongly associated with risk of lip cancer. J Intern Med 282: 322-331.

34. Shin D, Lee ES, Kim J, Guerra L, Naik D, et al. (2019) Association between the use of thiazide diuretics and the risk of skin cancers: A meta-analysis of observational studies. J Clin Med Res 11: 247-255. 